\title{
Uptake of citrate-coated iron oxide nanoparticles into atherosclerotic lesions in mice occurs via accelerated transcytosis through plaque endothelial cells
}

\author{
Wolfram C. Poller ${ }^{1,6,7}(\bowtie)$, Evelyn Ramberger ${ }^{1}$, Philipp Boehm-Sturm ${ }^{2,3}$, Susanne Mueller, ${ }^{2,3}$, Konstantin Möller $^{1}$, \\ Norbert Löwa ${ }^{4}$, Frank Wiekhorst ${ }^{4}$, Susanne Wagner ${ }^{5}$, Matthias Taupitz ${ }^{5}$, Eyk Schellenberger ${ }^{5}$, Gert Baumann ${ }^{1,6}$, \\ Karl Stangl ${ }^{1,6}$, Verena Stangl ${ }^{1,6}(\bowtie)$, and Antje Ludwig ${ }^{1,6}$ \\ ${ }^{1}$ Medizinische Klinik mit Schwerpunkt Kardiologie und Angiologie, Charité-Universitätsmedizin Berlin, Campus Mitte, Charitéplatz 1, \\ 10117 Berlin, Germany \\ ${ }^{2}$ Abteilung für Experimentelle Neurologie, Center for Stroke Research, Charité-Universitätsmedizin Berlin, Charitéplatz 1, 10117 \\ Berlin, Germany \\ ${ }^{3}$ Charité Core Facility "7 T experimental MRIs", Charité-Universitätsmedizin Berlin, Charitéplatz 1, 10117 Berlin, Germany \\ ${ }^{4}$ Physikalisch-Technische Bundesanstalt, Abbestr. 2-12, 10587 Berlin, Germany \\ ${ }^{5}$ Institut für Radiologie, Charité-Universitätsmedizin Berlin, Campus Mitte, Charitéplatz 1, 10117 Berlin, Germany \\ ${ }^{6}$ DZHK (German Centre for Cardiovascular Research), partner site Berlin, 10115 Berlin, Germany \\ ${ }^{7}$ Berlin Institute of Health (BIH), 10117 Berlin, Germany
}

Received: 23 March 2016

Revised: 15 July 2016

Accepted: 17 July 206

(C) Tsinghua University Press and Springer-Verlag Berlin Heidelberg 2016

\section{KEYWORDS}

atherosclerosis, unstable plaques, magnetic resonance imaging, decreased endothelial barrier function, superparamagnetic iron oxide nanoparticles

\begin{abstract}
Very small superparamagnetic iron oxide nanoparticles (VSOPs) rapidly accumulate in atherosclerotic lesions, thereby enabling plaque visualization by magnetic resonance imaging (MRI). This study was performed to identify the uptake mechanisms of VSOPs into atherosclerotic plaques. Low-density lipoprotein receptor-deficient $\left(\mathrm{LDLR}^{--}\right)$mice with advanced atherosclerosis were analyzed using MRI and transmission electron microscopy (TEM) at various time points after intravenous administration of VSOPs. Post-mortem MRI detected VSOP labeling of atherosclerotic plaques $10 \mathrm{~min}$ after injection, and the signal increased over the first $3 \mathrm{~h}$. TEM revealed that the intensive plaque labeling was mediated by accelerated transcytosis of VSOPs through endothelial cells overlaying atherosclerotic lesions. Experiments with endocytosis inhibitors and small interfering RNA (siRNA) revealed a dynamin-dependent mechanism involving both clathrin- and caveolin-mediated processes. In cell culture experiments, endothelial VSOP uptake was enhanced under proatherogenic flow and TNF $\alpha$ stimulation, conditions that are both present in plaque areas. Our study demonstrates that VSOPs enable non-invasive MRI assessment of accelerated endothelial transcytosis, an important pathomechanism in atherosclerotic plaque formation.
\end{abstract}

Address correspondence to Wolfram Poller, wolfram.poller@charite.de; Verena Stangl, verena.stangl@charite.de 


\section{Introduction}

Atherosclerosis is the leading cause of death in human society [1]. Endothelial dysfunction promotes extravasation of lipids and leucocytes, proliferation of smooth muscle cells, and finally the formation of atherosclerotic plaques [2,3]. Decreased endothelial barrier function is a risk factor for plaque destabilization and rupture, with potentially life-threatening complications $[4,5]$. Noninvasive identification of unstable plaques prior to rupture is of great clinical importance [5]. Magnetic resonance imaging (MRI) with targetspecific contrast agents enables visualization of specific structures and processes in atherosclerotic plaques [6]. Whereas gadolinium-based contrast agents have been used for visualization of extracellular matrix components and endothelial permeability [7-10], superparamagnetic iron oxide nanoparticles (SPIOs) have been shown to label plaque macrophages as markers of inflammation [11, 12]. The strongest and fastest plaque labeling has been achieved with a certain type of SPIOs, so-called very small superparamagnetic iron oxide nanoparticles (VSOPs) [13-15]. Compared to conventional polymer-coated SPIOs, VSOPs are significantly smaller $(7 \mathrm{~nm})$ and coated with monomeric citrate, and they are thereby stabilized electrostatically [13]. In vitro experiments in THP-1 monocytic cells demonstrated that the rapid cellular VSOP uptake depends on an interaction with cell surface glycosaminoglycans (GAGs) [15]. In vivo MRI studies in apolipoprotein E-deficient $\left(\mathrm{ApoE}^{--}\right)$mice revealed an increasing uptake of VSOPs into atherosclerotic lesions over the course of disease progression and identified plaque macrophages as imaging targets [16, 17]. Plaque analyses in atherosclerotic rabbits confirmed VSOP accumulation in macrophages and uncovered GAGcontaining microvesicles as additional imaging targets [18]. In agreement with these instability-associated targets, the loss of MRI signal caused by VSOPs was reported to correlate with histological plaque instability criteria [18]. Recently, a study comparing VSOP variants stabilized with different monomeric organic acids detected VSOP-filled vesicular structures in the cytoplasm of plaque endothelial cells (ECs) $3 \mathrm{~h}$ after VSOP injection [17]. This observation adds an important new aspect to the discussion on potential VSOP uptake mechanisms. Although several studies have analyzed the imaging potential and target structures of VSOPs, the mechanisms underlying their rapid and strong accumulation in plaques remain unknown. Several pathways have been discussed, including uptake by blood monocytes with subsequent migration of labeled cells into the plaque, extravasation via leaky neovessels, and transport through plaque endothelium with decreased barrier function [19]. Decreased barrier function comprises intercellular pathways such as widening of tight junctions and partial or complete denudation, as well as transcellular pathways such as transcytosis $[2,20,21]$. The present study investigates the uptake mechanisms of VSOPs into atherosclerotic plaques of low-density lipoprotein receptor-deficient $\left(\mathrm{LDLR}^{--}\right.$) mice. VSOP-based MRI was correlated with electron microscopic analyses of VSOP uptake into plaques at various time points after VSOP injection.

\section{Results and discussion}

\subsection{MRI analyses of VSOP accumulation in atherosclerotic plaques}

The uptake mechanisms underlying the rapid accumulation of VSOPs in plaques were analyzed by combining MRI and transmission electron microscopy (TEM) in $\mathrm{LDLR}^{--}$mice with advanced atherosclerosis. We recorded T2*-weighted in situ and ex vivo MRI scans of the thoracic aorta at time points ranging from $10 \mathrm{~min}$ to $24 \mathrm{~h}$ after intravenous injection of VSOPs. VSOPs are rapidly cleared from the blood, mainly by liver and spleen, with a blood half-life of around $30 \mathrm{~min}$ [17], indicating that early time points after injection are the most important for uptake analyses. In vivo MR imaging of VSOP accumulation in plaques is difficult within the first two blood halflives, since during that period the high intravascular VSOP concentration has a strong $\mathrm{T}^{*}$ effect that prevents adequate visualization of the vessel wall [22]. Furthermore, high-resolution three-dimensional (3D) MR imaging of the aorta takes about $30 \mathrm{~min}$, which prohibits analyses of clearly defined time points in vivo. Therefore, we chose a post-mortem in situ approach to visualize sites of VSOP accumulation in the anatomical context and combined it with ex vivo 
scans that allowed quantification of the MRI signal in plaques at defined time points after particle injection. Administration of VSOPs caused significant signal loss within atherosclerotic plaques that were present at typical predilection sites such as the aortic root, the inner curvature of the aortic arch, and the brachiocephalic trunk (Figs. 1(a) and 1(b)). Crosssections of the aorta confirmed that signal extinctions caused by VSOPs were clearly located within atherosclerotic plaques in the vessel wall (Fig. 1(c)). No signal decay was observed in non-plaque areas of the vessel wall. In ex vivo scans, VSOP signals within plaques were clearly distinguishable from signal extinctions at the outside of the aorta that were caused by air bubbles generated during the agarose embedding process (Figs. 1(b) and 1(c)).
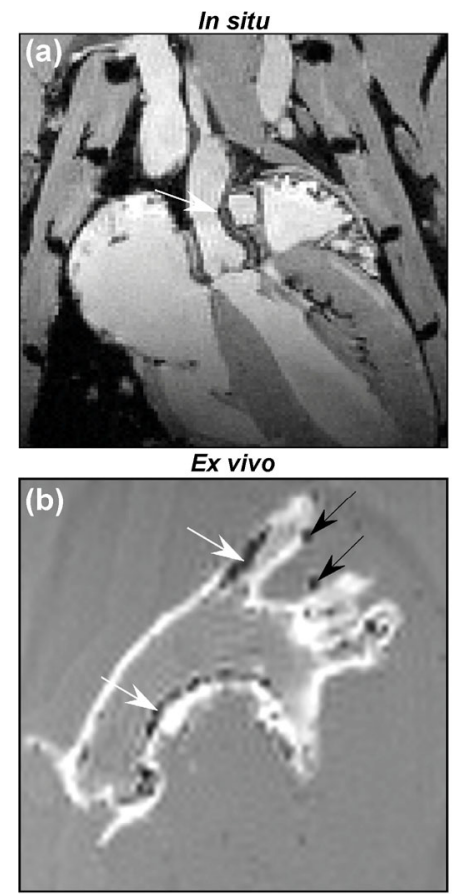

(d)

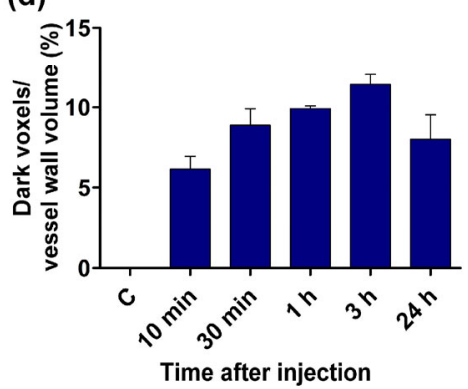

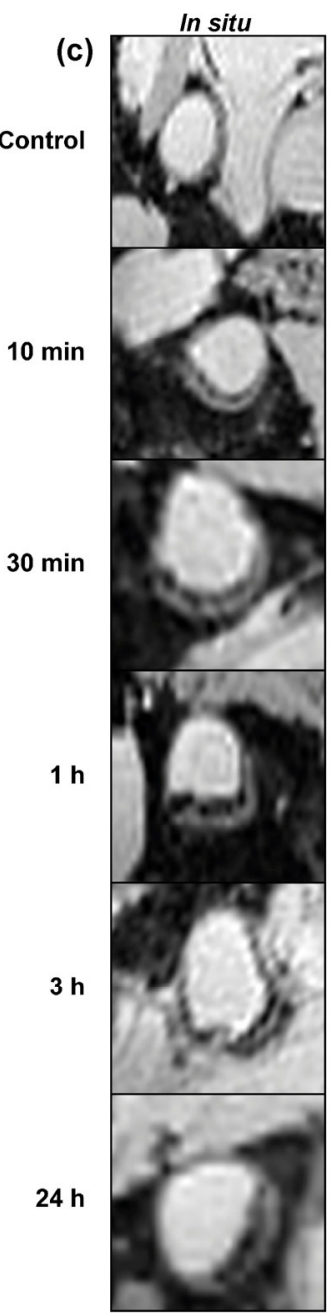

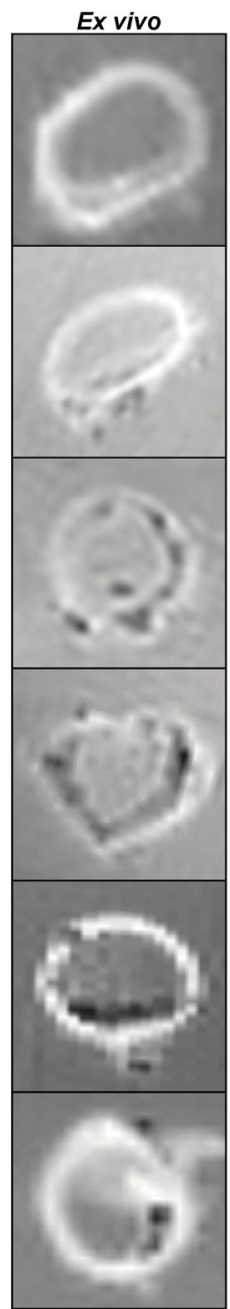

Figure 1 T2*-weighted magnetic resonance imaging scans of the thoracic aorta of low-density lipoprotein receptor-deficient $\left(\mathrm{LDLR}^{-/}\right)$mice at various time points after very small superparamagnetic iron oxide nanoparticle (VSOP) injection $\left(300 \mu \mathrm{mol} \cdot \mathrm{kg}^{-1}\right)$. (a) Post-mortem in situ MRI of the ascending aorta and aortic arch $3 \mathrm{~h}$ after VSOP injection. (b) Ex vivo MRI of the ascending aorta and aortic arch $3 \mathrm{~h}$ after VSOP injection. VSOP accumulation caused significant signal extinctions within atherosclerotic plaques (white arrows). Signal extinctions outside the aorta resemble air bubbles originating from agarose embedding (black arrows). (c) Examples of T2*-weighted in situ and ex vivo MRI cross-sections of the ascending aorta at the indicated time points after VSOP injection. VSOP deposits within atherosclerotic lesions are clearly discernable as signal extinctions in the vessel wall. (d) Quantification of VSOP uptake into aortic plaques of $\mathrm{LDLR}^{-/-}$mice at various time points after VSOP injection. The percentage of dark voxels in the vessel wall was determined in five consecutive ex vivo MRI cross-sections of the ascending aorta for each time point (data expressed as mean \pm standard deviation (SD)). VSOP accumulation in plaques was detectable by post-mortem MRI as early as 10 min after injection, with increasing signal intensities over the first $3 \mathrm{~h}$ and a slight decrease toward $24 \mathrm{~h}$. 
For semiquantitative analyses of VSOP uptake into plaques of the ascending aorta, the number of dark voxels was determined in 5 ex vivo aortic MRI cross-sections per animal. To minimize the influence of varying plaque size among the animals on quantification, we calculated the percentage of dark voxels as a proportion of all vessel wall voxels (Fig. 1(d)). Quantification revealed that VSOP accumulation in atherosclerotic lesions of $\mathrm{LDLR}^{-/}$mice was detectable by MRI as early as $10 \mathrm{~min}$ after injection, with increasing signal intensities over the first $3 \mathrm{~h}$. These results demonstrate that VSOPs were rapidly taken up into atherosclerotic lesions of $\mathrm{LDLR}^{-/-}$mice, causing clear MRI signals within plaques $10 \mathrm{~min}$ after injection. It must be pointed out that the results obtained from this ex vivo approach do not allow conclusions to be made regarding the in vivo imaging characteristics of VSOPs. Future experiments will be necessary to assess the optimal time frame for in vivo imaging.

\subsection{Analyses of the mechanisms of VSOP uptake into atherosclerotic plaques}

Extensive TEM analysis of aortic cross-sections of VSOP-treated $\mathrm{LDLR}^{-/}$mice allowed detailed investigation of VSOP uptake and accumulation in atherosclerotic plaques between $10 \mathrm{~min}$ and $24 \mathrm{~h}$ after injection (Fig. 2). For this approach, an appropriate fixation of atherosclerotic arteries for TEM was critical to preserve the ultrastructure of the inhomogeneous and lipid-rich plaques and to avoid artificial injury of the diseased endothelium. In a series of pre-tests, we observed a strong correlation between the extent of endothelial damage and the time from animal euthanasia to fixation. The best results with minimal artificial destruction of the vessel structure were achieved by direct transcardiac pressure-perfusion with a TEM-optimized fixation solution (see methods).

In the ascending aortas of animals that were analyzed within $1 \mathrm{~h}$ after injection, numerous small VSOP aggregates consisting of 3-15 individual nanoparticles were detectable in close proximity to the plaque endothelial surface (Figs. 2(a)-2(c)). Moreover, we frequently observed flask-shaped membrane invaginations with diameters of $50-80 \mathrm{~nm}$ filled with VSOPs at the luminal site of the endothelium (Figs. 2(d)-2(f)).
VSOP-containing invaginations subsequently fully engulfed the nanoparticles and formed small vesicles after scission from the plasma membrane (Fig. 2(g)). At $10 \mathrm{~min}$ after injection, small VSOP-filled vesicles were already visible in the cytoplasm, and they later fused to form larger vesicles containing hundreds of nanoparticles (Figs. 2(h) and 2(i)). Eventually, VSOP aggregates appeared in the subendothelial space (Figs. 2(j)-2(1)). Based on these observations, we concluded that VSOPs were delivered across ECs via transcytosis.

It was previously suggested that VSOP-labeled blood-born monocytes are recruited into inflamed atherosclerotic areas of $\mathrm{ApoE}^{-/-}$mice, since macrophages have been identified as the main site of VSOP accumulation within plaques [16]. Our TEM analysis revealed that VSOPs were indeed rapidly and efficiently taken up by peripheral blood mononuclear cells (PBMCs) in the circulation of $\mathrm{LDLR}^{-/}$mice (Figs. 3(a) and 3(b)). Considerable amounts of internalized VSOPs were already detectable in murine PBMCs at $10 \mathrm{~min}$ after injection. PBMC-associated VSOPs were mainly located in vesicles with diameters of 100-250 nm, each containing hundreds of VSOPs (Fig. 3(a)). A decrease in the number of circulating VSOP-positive PBMCs was measured $3 \mathrm{~h}$ after injection. Although we did not observe any transmigration of VSOP-labeled PBMCs into atherosclerotic plaques by TEM, this uptake mechanism cannot be ruled out. Since considerable numbers of circulating VSOP-loaded PBMCs were persistently detectable up to $7 \mathrm{~d}$ after injection (Fig. 3(b)), it is conceivable that these cells may gradually contribute to the labeling of atherosclerotic lesions with superparamagnetic iron. In contrast to PBMCs, VSOPs were not detected in red blood cells (RBCs) isolated from VSOP-injected $\mathrm{LDLR}^{-/}$mice (Fig. 3(c)). In addition, we compared the uptake of VSOPs into isolated PBMCs and RBCs in vitro using the highly sensitive superparamagnetic nanoparticlespecific magnetic particle spectroscopy (MPS). As shown in Table 1, superparamagnetic iron was already detectable inside PBMCs after 10 min of incubation with $0.75 \mathrm{mM}$ VSOPs, and the intracellular concentration increased with longer incubation time. In contrast, the superparamagnetic iron content in RBC samples was below the detection limit of MPS (limit 


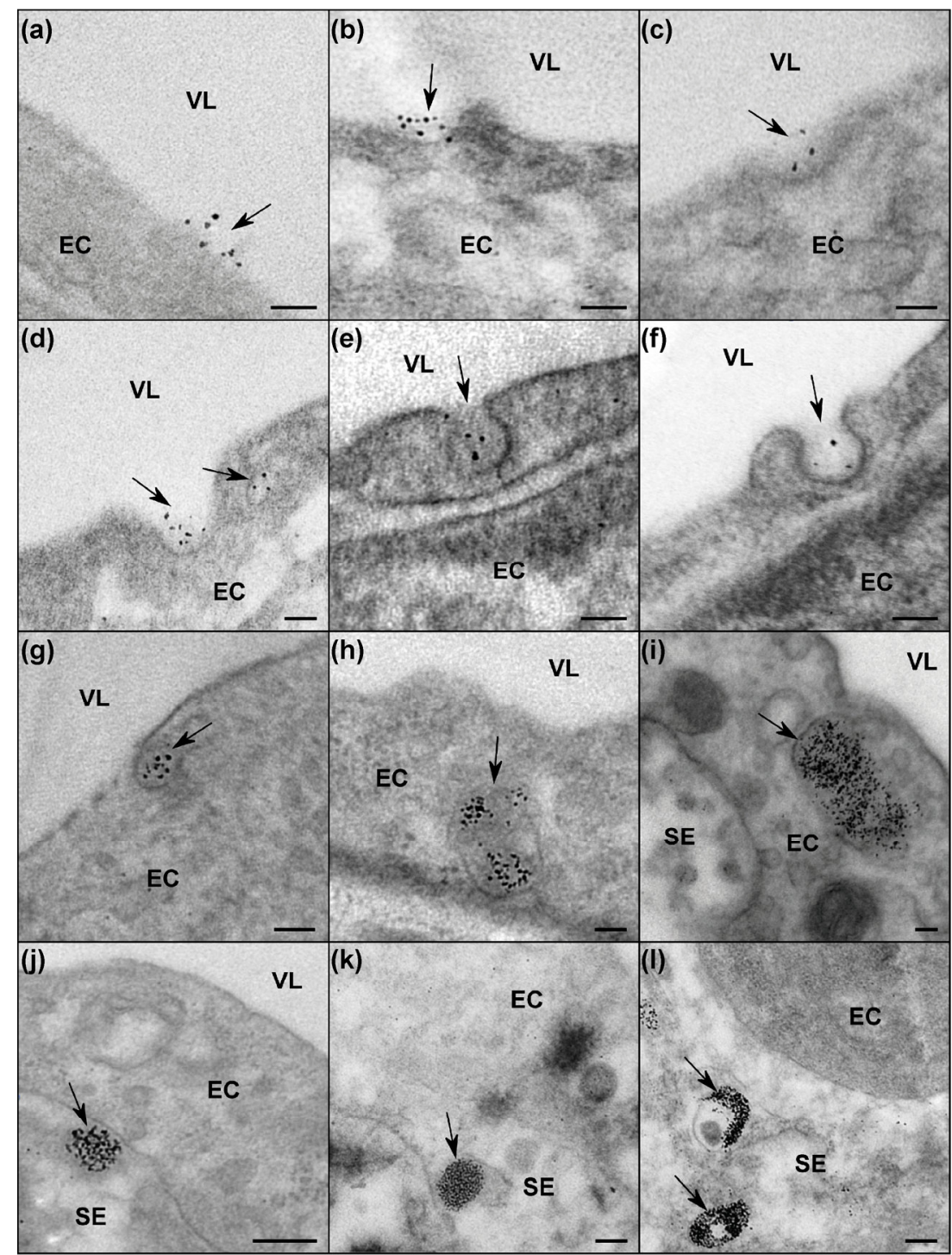

Figure 2 TEM analyses of VSOP uptake into aortic atherosclerotic lesions of $\mathrm{LDLR}^{-/-}$mice $10 \mathrm{~min}$ to $24 \mathrm{~h}$ after injection. VSOPs are visible as electron-dense spots with diameters of $\sim 7 \mathrm{~nm}$ (arrows). (a) Initial binding of small VSOP aggregates to the luminal surface of ECs. (b) and (c) VSOPs associated with invaginations of the plasma membrane. (d)-(f) Endocytic pits containing nanoparticles. (g) Scission of a VSOP-containing pit from the plasma membrane. (h) and (i) Fusion of small VSOP-filled vesicles to larger vesicles. (j)-(l) VSOP aggregates in the subendothelial space of atherosclerotic lesions. Time after injection: (a)-(e) $10 \mathrm{~min}$; (f)-(h) $30 \mathrm{~min}$; (i) $3 \mathrm{~h}$; (j)-(l) 24 h. Scale bars: (a)-(i) $50 \mathrm{~nm}$; (j)-(l) $100 \mathrm{~nm}$. VL—vascular lumen, EC—endothelial cells, SE—subendothelial space.

of detection $=91 \mathrm{ng})$, even after $1 \mathrm{~h}$ of incubation with VSOPs (Table 1). Since RBCs are incapable of endocytosis [23], we concluded that the cellular uptake of VSOPs strictly depends on endocytic processes. Passive membrane penetration as an uptake mechanism for VSOPs is not supported by our data.

In our detailed TEM studies, we did not observe the passage of VSOPs via intercellular pathways through interendothelial junctions (IEJs) or regions of endothelial denudation. Having a hydrodynamic diameter of $8.7-11 \mathrm{~nm}$, VSOPs are too large to pass through intact IEJs. Interestingly, the frequent occurrence of widened IEJs, as reported for $\mathrm{ApoE}^{-/-}$mice with advanced atherosclerosis, could not be confirmed in our experimental setup using the present fixation protocol [10]. Areas of endothelial denudation with exposure of subendothelial structures were rarely observed, and no VSOP accumulation was detectable in these regions. 


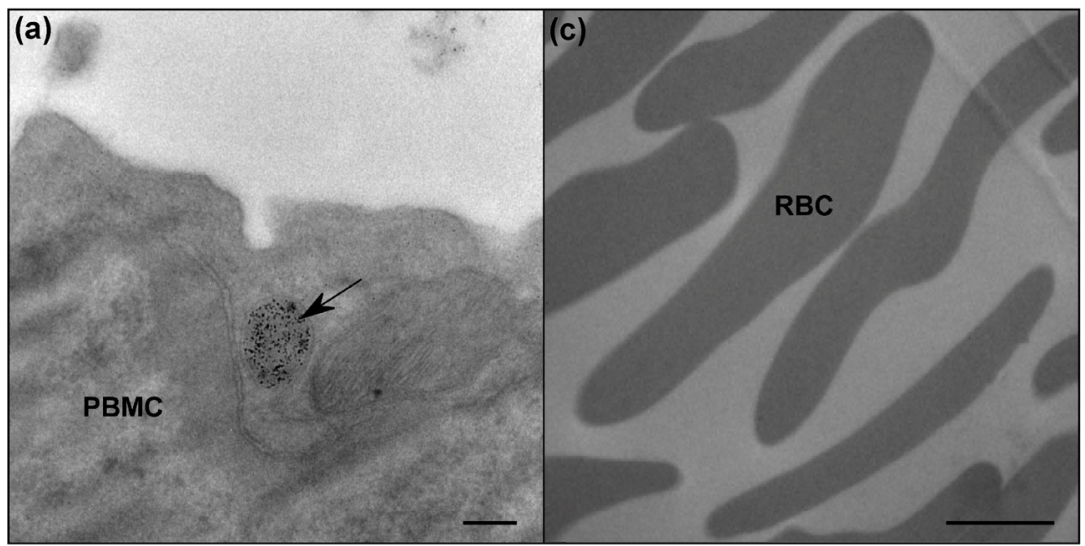

(b)

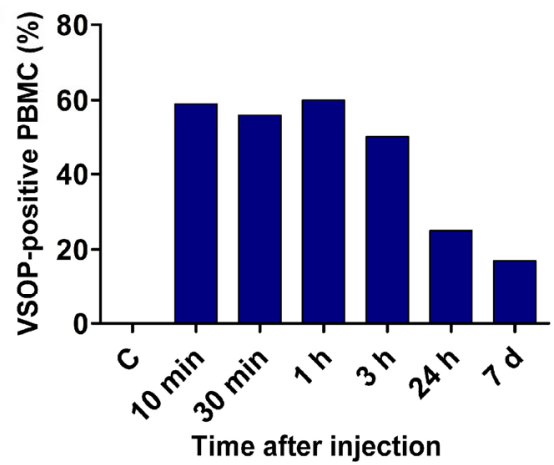

Figure 3 TEM analyses of in vivo VSOP uptake into peripheral blood mononuclear cells (PBMCs) and red blood cells (RBCs) of LDLR $^{-/-}$mice. (a) PBMC with a VSOP-filled cytoplasmic vesicle $3 \mathrm{~h}$ after VSOP injection (arrow). (b) Percentage of VSOP-positive PBMCs determined by TEM analyses of $\geq 100$ PBMCs for each time point after injection. The percentage of VSOP-positive PBMCs remained at high levels up to $3 \mathrm{~h}$ after injection. Even at $7 \mathrm{~d}$ after injection, VSOP-positive PBMCs were still detectable. (c) RBCs isolated from $\mathrm{LDLR}^{-/-}$mice $3 \mathrm{~h}$ after VSOP injection did not contain VSOPs. Scale bars: (a) $100 \mathrm{~nm}$; (c) $1 \mu \mathrm{m}$.

Table 1 Comparison of VSOP uptake into peripheral blood mononuclear cells and red blood cells in vitro

\begin{tabular}{ccc}
\hline $\begin{array}{c}\text { VSOP } \\
\text { incubation time }\end{array}$ & $\begin{array}{c}\mathrm{PBMCs}^{\mathrm{a}} \\
\mathrm{cFe}_{\mathrm{MPS}}{ }^{\mathrm{C}}(\mathrm{pg} / \text { cell })\end{array}$ & $\begin{array}{c}\mathrm{RBCs}^{\mathrm{b}} \\
\mathrm{cFe}_{\mathrm{MPS}}{ }^{\mathrm{d}}(\mathrm{pg} / \mathrm{cell})\end{array}$ \\
\hline 0 & n.d. $^{\mathrm{d}}$ & n.d. $^{\mathrm{d}}$ \\
$10 \mathrm{~min}$ & $0.62 \pm 0.10$ & n.d. $^{\mathrm{d}}$ \\
$30 \mathrm{~min}$ & $1.17 \pm 0.12$ & n.d. $^{\mathrm{d}}$ \\
$1 \mathrm{~h}$ & $1.34 \pm 0.07$ & n.d. $^{\mathrm{d}}$ \\
\hline
\end{tabular}

${ }^{\mathrm{a}} \mathrm{PBMCs}=$ peripheral blood mononuclear cells isolated from $\mathrm{LDLR}^{-/-}$mice; ${ }^{\mathrm{b}} \mathrm{RBCs}=$ red blood cells isolated from $\mathrm{LDLR}^{-/-}$ mice; ${ }^{\mathrm{c}} \mathrm{CF} \mathrm{MPS}_{\mathrm{MP}}=$ concentration of superparamagnetic iron measured by magnetic particle spectroscopy; data are expressed as mean \pm standard deviation, $n=3$, ${ }^{\mathrm{d}}$.d. $=$ non-detectable.

Therefore, we assumed that the observed denudation probably occurred artificially during the preparation procedure.

In our experiments, we did not observe neovessels within plaques of $\mathrm{LDLR}^{-/-}$mice. Neovascularization mediated by hypoxia occurs when the tunica intima thickens to a width of above $500 \mu \mathrm{m}$ [24]. Hence, the shorter diffusion distances in mouse plaques compared to those in human plaques may explain the absence of neovessels in our model. The extravasation of VSOPs via neovessels in humans or other species cannot be excluded by our study. However, rapid and efficient plaque labeling with VSOPs obviously does not depend on the presence of neovessels.

We concluded that uptake by blood monocytes with subsequent migration of labeled cells into the plaque, transport via intercellular pathways, and delivery via neovessels do not considerably account for the rapid VSOP enrichment in plaques that enables MRI within $1 \mathrm{~h}$ after injection. Our current data provide evidence for endothelial transcytosis as the main uptake mechanism of VSOPs into atherosclerotic plaques of $\mathrm{LDLR}^{-/-}$mice.

In a following step, we investigated the endocytic mechanisms mediating VSOP uptake into ECs in greater detail by performing in vitro experiments with 
human umbilical vein ECs (HUVECs). First, HUVECs were treated with dynasore, a specific inhibitor of the GTPase dynamin, which is essential for scission of newly formed endocytic vesicles from the plasma membrane in clathrin- and caveolin-dependent endocytosis [25]. We used dynasore at concentrations of
75 and $150 \mu \mathrm{M}$, which were sufficient to inhibit the internalization of fluorescence-labeled bovine serum albumin (BSA) and human transferrin (hTF), both of which are well-established markers for dynamindependent endocytic processes (Fig. 4(a)). The efficient uptake of VSOPs into HUVECs was significantly

(a)

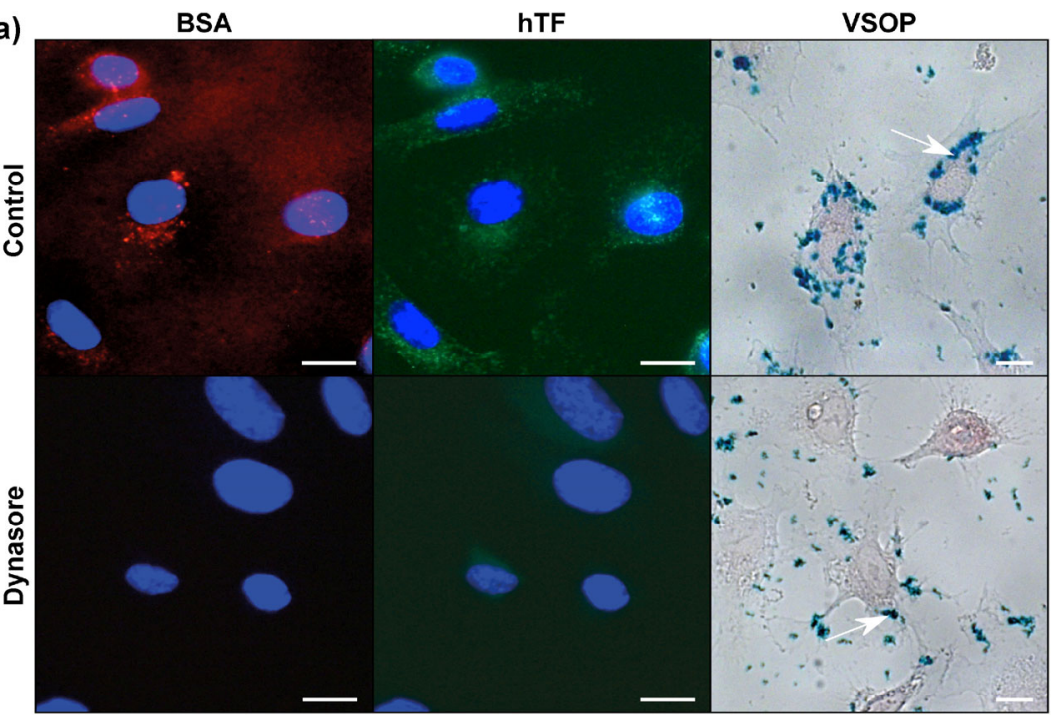

(b)

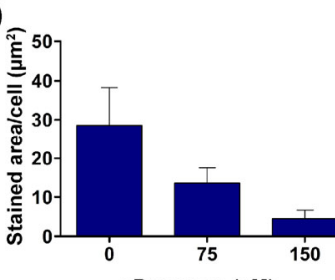

(c) SCR Si-CLT

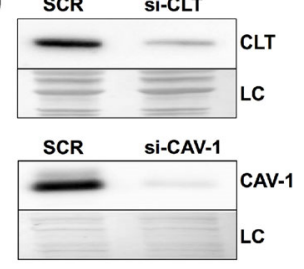

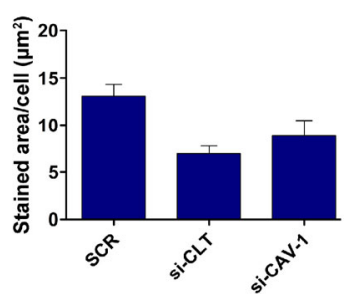

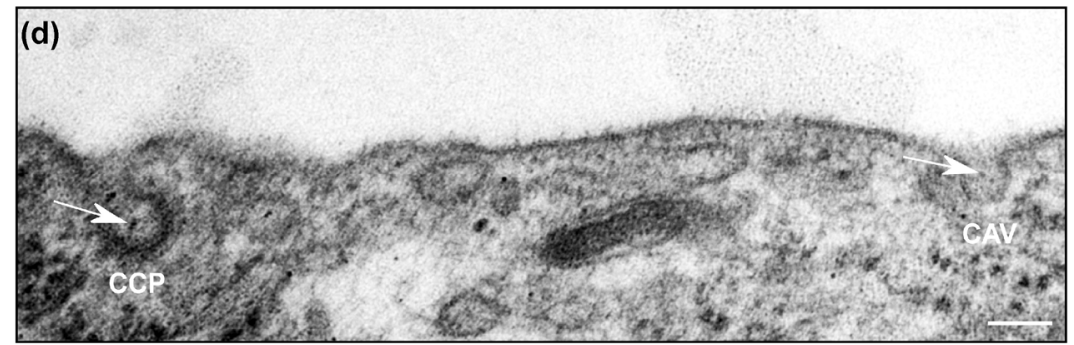

Figure 4 VSOP uptake is dynamin-dependent and involves clathrin- and caveolin-mediated processes. (a) HUVECs were grown on chambered coverslips. After pretreatment with vehicle (upper panel) or $75 \mu \mathrm{M}$ dynasore for 30 min to inhibit dynamin function (lower panel), cells were incubated with Texas Red-labeled bovine serum albumin (BSA), Fluorescein isothiocyanate (FITC)-labeled human transferrin (hTF), or VSOPs for $60 \mathrm{~min}$. Internalized BSA-Texas Red and hTF-FITC were visualized by fluorescence microscopy, VSOPs were visualized by light microscopy after Prussian blue staining. (b) Quantification of VSOP uptake into HUVECs after pretreatment with 75 and $150 \mu \mathrm{M}$ dynasore by measurement of the Prussian blue-stained area per cell compared with that of vehicletreated controls. (c) Immunoblots showing clathrin heavy chain (CLT) and caveolin-1 protein (CAV-1) levels after knockdown with siRNA in HUVECs and quantification of VSOP uptake into HUVECs after knockdown of CLT and CAV-1 compared with control siRNA (SCR). Amido black staining of immunoblot membranes served as a control for equal protein loading (LC). Data are presented as mean $\pm \mathrm{SD}, n=3$ experiments. (d) TEM image of an aortic plaque EC of a VSOP-injected LDLR ${ }^{-/-}$mouse after post-contrasting with uranyl acetate and lead citrate showing VSOPs (arrows) associated with a clathrin-coated pit (CCP) and a caveolae-like structure (CAV). Scale bars: (a) $10 \mu \mathrm{m}$; (d) $100 \mathrm{~nm}$. 
inhibited by dynasore (Figs. 4(a) and 4(b)). Knockdown of caveolin-1 (CAV-1) or clathrin heavy chain protein (CLT) with small interfering RNA (siRNA) in HUVECs resulted in decreased VSOP uptake (Fig. 4(c)). These experimental data indicate that VSOP uptake into ECs is dynamin-dependent, involving both caveolinand clathrin-mediated processes. We verified the existence of VSOP-engulfing clathrin-coated pits and caveolae in the endothelium of $\mathrm{LDLR}^{-/-}$mice by TEM analyses of post-contrasted aortic cross-sections (Fig. 4(d)).

\subsection{Comparison of VSOP uptake into plaque and non-plaque ECs}

Figure 5(a) shows the characteristic histology of aortic arch cross-sections of $\mathrm{LDLR}^{-/}$mice. Due to flow conditions, atherosclerotic lesions were mainly located in the inner curvature of the aortic arch, whereas the outer curvature exhibited mostly non-diseased vessel wall. During TEM analyses, we differentiated between plaque ECs and non-plaque ECs based on their morphologies: ECs covering subendothelial foam cells and extracellular matrix deposits were defined as plaque ECs (Fig. 5(b)), whereas ECs covering vessel areas with intact subendothelial layers including the internal elastic lamina and basement membrane were defined as non-plaque ECs (Fig. 5(c)). We analyzed 200 plaque ECs and 200 non-plaque ECs at each time point after VSOP injection. As depicted in Fig. 5(d), the percentage of VSOP-positive ECs and the number of VSOP-filled vesicles per EC were significantly higher in plaque ECs than in non-plaque ECs at all time points and reached a maximum at $30 \mathrm{~min}$ after injection (paired $t$-test, $p<0.01$ ). The lower number of VSOP-filled vesicles at later time points can be explained by the decreased plasma concentration of VSOPs, which minimized further uptake from the vessel lumen, the fusion of small vesicles to larger vesicles in the cytoplasm of ECs, and the subsequent release of VSOPs into the subendothelial space. Accumulations of VSOPs in subendothelial areas and foam cells were exclusively observed in atherosclerotic lesions. Our TEM analyses demonstrate that the specific enrichment of VSOPs in atherosclerotic lesions is attributed to an increased transcytosis of nanoparticles through plaque ECs. Thus, early signals in VSOP-based MRI reflect the increase in endothelial transcytosis within atherosclerotic plaques.

Visualization of increased endothelial permeability is of great clinical relevance, e.g., for the detection of unstable plaques and for the monitoring of interventions that aim to restore the endothelium. Several imaging studies with various contrast agents, e.g., albuminbinding gadofosveset and fluorescence-labeled liposomal nanoparticles, have suggested that increased endothelial permeability is important for agent uptake and plaque visualization [10, 26]. Increased endothelial permeability is a collective term that comprises several mechanisms, including the widening of IEJs, gap formation, erosion and denudation, and various types of vesicular transcytosis. To the best of our knowledge, we are the first to describe accelerated endothelial transcytosis within atherosclerotic plaques as an imaging target for MRI. Most importantly, accelerated endothelial transcytosis is an important pathomechanism that is considered to be a major route for low-density lipoprotein (LDL) uptake into atherosclerotic plaques $[27,28]$.

\subsection{Evaluation of processes that accelerate endocytosis of VSOPs into ECs}

Endothelial function is substantially determined by the continuous apical shear forces produced by flowing blood. Disturbed laminar blood flow in vessel curves and bifurcations contributes to endothelial dysfunction that accelerates atherogenesis at these sites [29]. We tested the hypothesis that flow conditions modulate the uptake of VSOPs into cultivated HUVECs. As expected, laminar flow altered cell morphology: HUVECs cultivated under laminar flow were aligned parallel to the direction of the flow, while cells cultivated in the absence of laminar flow were diffusely distributed (Fig. 6(a)). HUVECs cultivated under laminar flow conditions only marginally internalized VSOPs (Figs. 6(a) and 6(b)). Stimulation with tumor necrosis factor (TNF) $\alpha$ caused a significant increase of VSOP uptake into HUVEC cultivated under laminar flow (25-fold higher than in non-stimulated HUVECs) (Figs. 6(a) and 6(b)). Remarkably, VSOP uptake was 


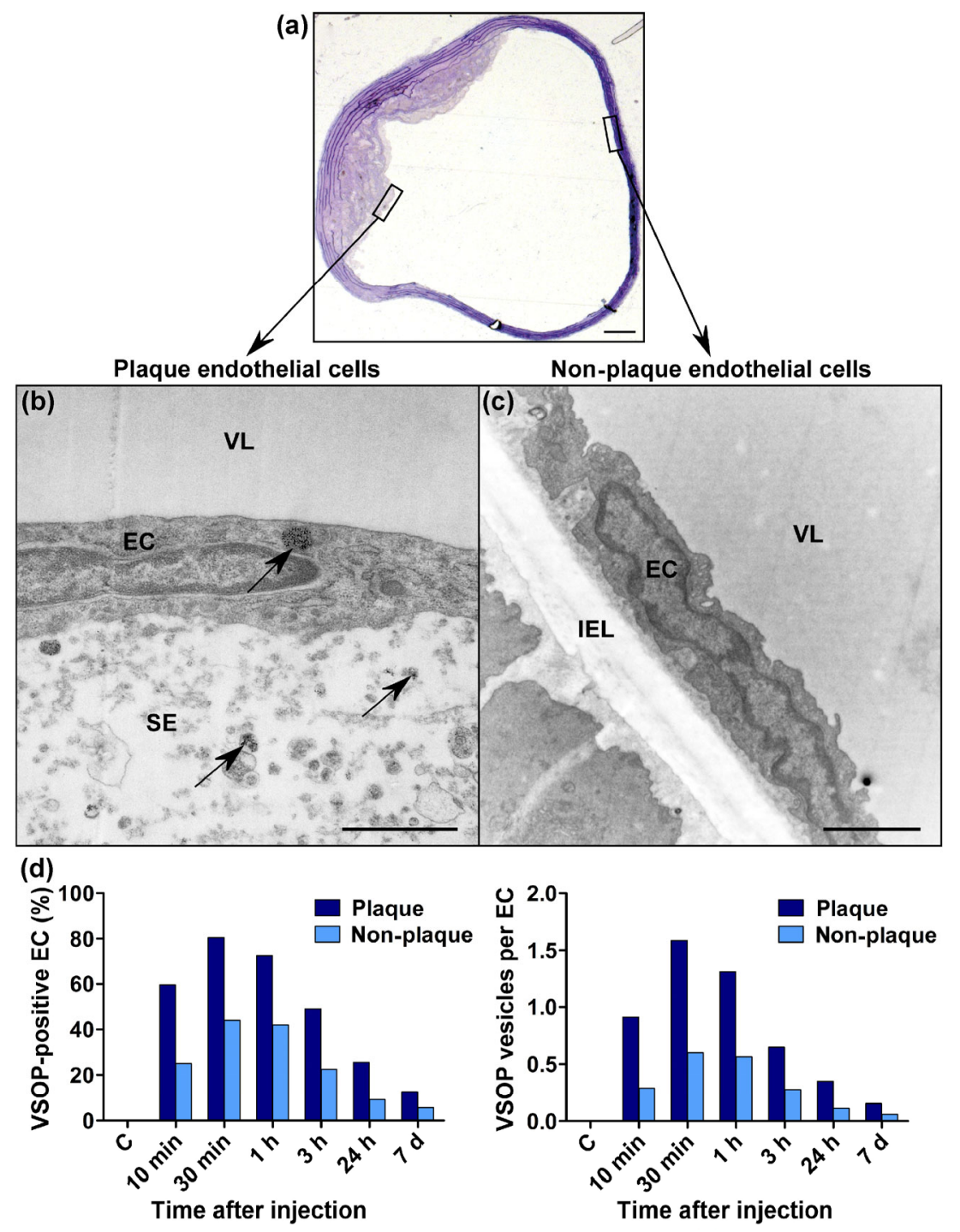

Figure 5 Comparison of VSOP uptake into plaque and non-plaque ECs of LDLR ${ }^{-/}$mice. (a) Toluidine-blue stained semithin section $(500 \mathrm{~nm})$ of the aorta of an $\mathrm{LDLR}^{--}$mouse with an advanced-stage atherosclerotic plaque in the inner curvature of the aortic arch. (b) TEM image of a plaque EC covering foam cells and extracellular matrix deposits. VSOPs are indicated by arrows. (c) TEM image of a non-plaque EC with intact subendothelial layers. (d) The percentage of VSOP-positive ECs and the average number of vesicles per EC were significantly higher in plaque ECs than in non-plaque ECs at all time points. Data were acquired by TEM analyses of 200 plaque ECs and 200 non-plaque ECs for each time point after injection. Scale bars: (a) $100 \mu \mathrm{m}$; (b) and (c) 1,000 nm. VL—vascular lumen, EC — endothelial cells, SE—-subendothelial space, IEL—internal elastic lamina.

exceedingly high in HUVECs maintained under static conditions (48-fold higher than in HUVECs under laminar flow) (Figs. 6(a) and 6(b)). To study the differential effects of uniform laminar flow and disturbed flow on VSOP uptake, HUVECs were exposed to laminar flow in Y-shaped channels, which mimic the shear-stress conditions present at arterial bifurcations. HUVECs exposed to disturbed flow, as present at the branch and kink regions, showed a strongly increased uptake of VSOPs compared to cells exposed to uniform laminar shear stress, as present at the straight segments (Figs. 6(c) and 6(d)).

These results demonstrate that inflammation and disturbed laminar flow, two factors that play major roles in endothelial dysfunction, contribute to the accelerated endocytosis of VSOPs into the plaque endothelium.

As a possible mechanism, we considered the 


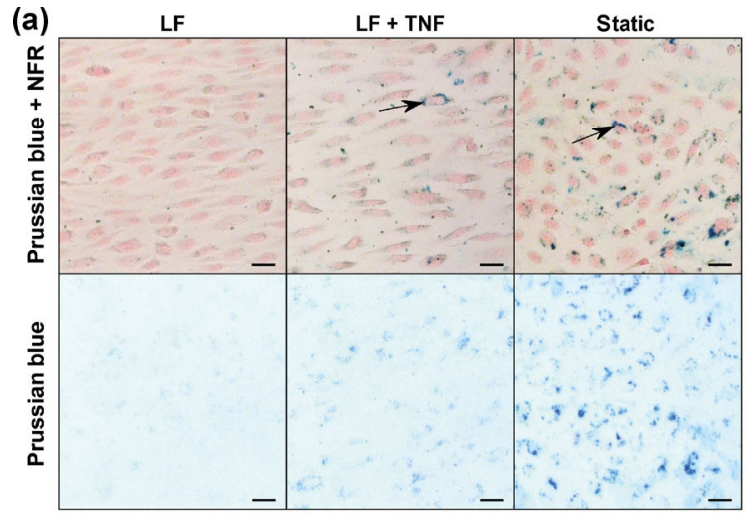

(c)

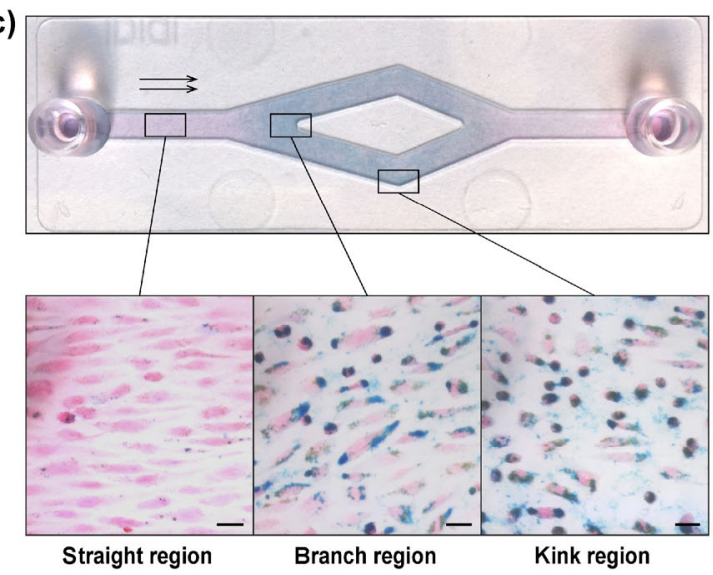

(b)

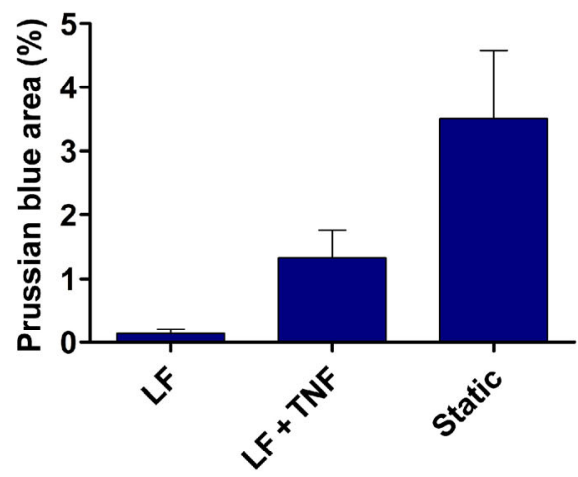

(d)

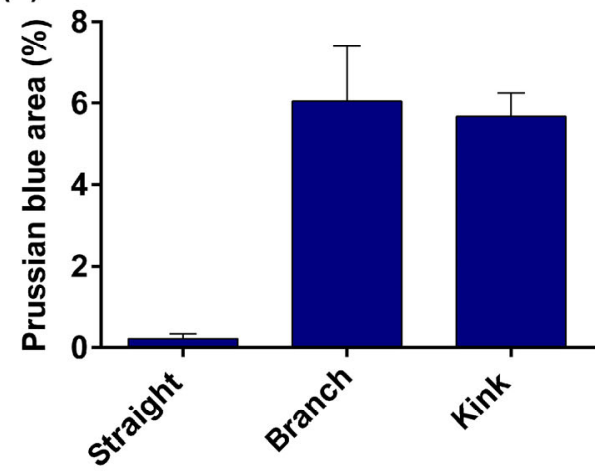

Figure 6 Effects of shear forces and pro-inflammatory stimulation on VSOP uptake into HUVECs. (a) Confluent cells grown on IbiTreat $\mu$-slides I 0.8 Luer were exposed to laminar flow (LF) for $72 \mathrm{~h}$ with or without stimulation with $5 \mathrm{ng} \cdot \mathrm{mL}^{-1} \mathrm{TNF}-\alpha(\mathrm{LF}$ and $\mathrm{LF}+$ TNF), or were grown under static conditions before the application of VSOPs for $3 \mathrm{~h}$ under LF. Nuclear fast red staining (NFR) revealed the typical parallel alignment of HUVECs to the direction of LF. VSOPs were detected by light microscopy after Prussian blue staining (arrows). (b) Quantification of VSOP uptake into HUVECs grown under LF, LF + TNF, and under static conditions by determination of the percentage Prussian blue stained area per region of interest (ROI). Data are presented as mean \pm SD; $n=7$ experiments, 6 ROI per treatment for each experiment. (c) Confluent HUVECs grown on IbiTreat $\mu$-slides Y-shaped were exposed to LF for $48 \mathrm{~h}$ before the application of VSOPs for $3 \mathrm{~h}$ under LF. Arrows indicate the direction of LF. VSOPs were detected by light microscopy after Prussian blue staining. Cell nuclei were visualized by NFR. (d) Quantification of VSOP uptake into HUVECs grown in areas with uniform laminar shear stress (straight segments) or in areas with disturbed flow (branch regions and kink regions) by determination of the percentage Prussian blue-stained area per ROI. Data are presented as mean $\pm \mathrm{SD} ; n=3$ experiments, 6 ROI per area (straight, branch, and kink) for each experiment. Scale bars: (a) and (c), $20 \mu \mathrm{m}$.

modulation of the glycocalyx of pathologically altered ECs. Indeed, TEM images of VSOP uptake into ECs in vivo showed that VSOPs initially do not bind directly to the EC membrane (Figs. 2(a) and 2(b)), but rather to the cell surface glycocalyx, a mesh of membraneassociated GAGs [30]. The high affinity of VSOPs to GAGs was reported in recent in vivo and in vitro studies [15, 18]. We hypothesized that the VSOP-GAG interaction occurs in a transchelation reaction in which the citrate coat of the cationic iron oxide core of the VSOPs is replaced by a GAG coat. This hypothesis was supported by the observation that the magnetic properties of VSOPs change immediately after contact with cells [31]. Considering that an electrostatic interaction with cell surface GAGs and subsequent endocytosis of the resulting complexes have been reported for polycationic macromolecules and cationic peptides [32], and for phagocytosis of VSOPs into macrophages [15], we propose a similar mechanism for the uptake of VSOPs into the endothelium. It must be considered that, after injection, nanoparticles adsorb a so-called biomolecule corona, which is a dynamic layer of proteins, lipids, and carbohydrates. A recent study showed that the GAG hyaluronan 
exhibits an exceptionally strong affinity toward citratecoated gold nanoparticles, which allows for effective competition with other serum proteins for adsorption to the gold nanoparticles [33]. The preferential binding of citrate-coated VSOPs to GAGs can therefore be expected, although it is probable that a considerable part of the nanoparticles initially interacts with other biomolecules in the blood.

It has previously been shown that inflammatory stimuli and disturbed laminar blood flow induce changes of the glycocalyx, not only by altering gene expression in ECs but also by modifying the 3D arrangement of proteoglycans and their associated GAGs on the EC surface [34,35]. Shear forces can influence the ability of the glycocalyx to recognize and retain extracellular ligands, since the conformation of the glycocalyx determines the accessibility of extracellular ligands to their binding partners on the cell surface [36]. To date, only a few studies have investigated the influence of flow on nanoparticle uptake into ECs [37-39]. Whether the uptake is inhibited [38] or enhanced [37] in response to laminar flow most probably depends on the binding partner on the cell surface and on the size and charge of the particles [40].

\section{Conclusion}

This study provides further evidence for the potential of VSOP for rapidly visualizing atherosclerotic lesions. We describe here for the first time the uptake process of VSOPs into atherosclerotic plaques. The rapid and efficient uptake of VSOPs is mediated by accelerated dynamin-dependent transcytosis through plaque ECs. Inflammation and disturbed laminar flow were identified as causes of the accelerated transcytosis. With these characteristics, VSOPs enable the noninvasive MRI assessment of increased endothelial transcytosis, an important pathomechanism in atherogenesis.

\section{Methods}

\subsection{Synthesis of VSOPs}

Chemicals and solvents were obtained from SigmaAldrich, St. Louis, MO, unless otherwise stated. For the synthesis of citrate-coated iron oxide nanoparticles, $14 \mathrm{~g}$ ferrous chloride tetrahydrate and $24.7 \mathrm{~g}$ ferric chloride hexahydrate were each dissolved in $100 \mathrm{~mL}$ deoxygenated $\mathrm{H}_{2} \mathrm{O}_{\text {bidest }}$ at $2{ }^{\circ} \mathrm{C}$ and then combined. To this solution, $90 \mathrm{~mL}$ of $28 \%$ ammonium hydroxide cooled to $2{ }^{\circ} \mathrm{C}$ was quickly added. The mixture was stirred for $1 \mathrm{~h}$ at $2{ }^{\circ} \mathrm{C}$ and magnetically sedimented $(0.5 \mathrm{~T})$. The supernatant was withdrawn and the sediment was dispersed with $300 \mathrm{~mL} 0.348 \mathrm{M}$ citric acid. This mixture was heated to $80-90{ }^{\circ} \mathrm{C}$ and stirred for $1 \mathrm{~h}$ at this temperature. After cooling to room temperature (RT), the mixture was again magnetically sedimented and the supernatant was centrifuged. The supernatant resulting from this centrifugation step was subsequently diluted and ultrafiltered (Vivaflow $^{\circledR}$, PES, $100 \mathrm{kDa}$ ) to a conductivity of the filtrate $<10 \mu \mathrm{S} \cdot \mathrm{m}^{-1}$. The retentate was diluted with $100 \mathrm{~mL} 0.25 \%(\mathrm{w} / \mathrm{v})$ sodium chloride solution and adjusted to a $\mathrm{pH}$ of 5.5 with citric acid, then ultrafiltered again. The retentate was diluted with $100 \mathrm{~mL} \mathrm{H}_{2} \mathrm{O}_{\text {bidest }}$ and ultrafiltered. This procedure was repeated until the filtrate reached a conductivity $<10 \mu \mathrm{S} \cdot \mathrm{m}^{-1}$. After iron quantification, the retentate was adjusted to a concentration of $0.15 \mathrm{M}$ Fe with $\mathrm{H}_{2} \mathrm{O}_{\text {bidest }}$. After adjusting to $\mathrm{pH} 7.0$ with $0.5 \mathrm{M}$ disodium citrate, the solution was formulated with mannitol (final concentration: $\left.60 \mathrm{~g} \cdot \mathrm{L}^{-1}\right)$. The final formulation was heat-sterilized. At a final iron concentration of $0.116 \mathrm{M}$, a citric acid concentration of $0.937 \mathrm{~g} \cdot \mathrm{L}^{-1}$ was measured. The mean hydrodynamic diameter of the nanoparticles (as measured by laser light scattering) was $8.7-11.0 \mathrm{~nm}$ with a polydispersity index of 0.085 , indicating a narrow distribution. Relaxivities in water were $r 1=$ $25 \mathrm{mM}^{-1} \cdot \mathrm{s}^{-1}$ and $r 2=63.8 \mathrm{mM}^{-1} \cdot \mathrm{s}^{-1}$ at $0.94 \mathrm{~T}$ at a saturation magnetization of $95 \mathrm{emu} \cdot \mathrm{g}^{-1}$ iron. The crystallite size measured by TEM (largest diameter of 500 crystals evaluated) was $6.8 \pm 2 \mathrm{~nm}$. The final ferrous iron ion content of $0.9 \%$ (molar ratio total iron) indicates nearly complete oxidation to maghemite. Selected area electron diffraction revealed a pure magnetite/maghemite pattern.

\subsection{Animals and treatments}

Animal experiments were approved by the local authority (Landesamt für Gesundheit und Soziales, Berlin) and were performed according to institutional 
guidelines. Animals were kept under standard housing conditions and a $12 \mathrm{~h}$ day/night cycle with water and food ad libitum. A total of 14 male 10-week-old LDLR ${ }^{-1}$ mice (B6.129S7-Ldlrtm1Her/J; JAX Mice, Boston) were fed a high-fat diet for 20 weeks ad libitum (Westerntype diet containing $21 \%(\mathrm{w} / \mathrm{w})$ butterfat, $17 \%(\mathrm{w} / \mathrm{w})$ casein, and $0.21 \%(\mathrm{w} / \mathrm{w})$ cholesterol; Ssniff, Soest, Germany). Animals received VSOPs at a dose of $300 \mu \mathrm{mol} \cdot \mathrm{kg}^{-1}$ of bodyweight via tail vein injection. Two animals were analyzed at each of the following time points after VSOP injection: $10 \mathrm{~min}, 30 \mathrm{~min}, 1 \mathrm{~h}$, $3 \mathrm{~h}, 24 \mathrm{~h}$, and $7 \mathrm{~d}$. Two animals without VSOP injection served as controls. Mice were sacrificed under isoflurane general anesthesia as described below. For in vitro uptake experiments, PBMCs and RBCs were isolated from $3 \mathrm{LDLR}^{-/-}$mice that were fed a normal diet.

\subsection{MRI}

For post-mortem in situ MRI analyses of the thoracic aorta, animals were sacrificed under isoflurane general anesthesia by blood withdrawal. The abdominal aorta was cannulated by retrograde puncture and the inferior vena cava was opened for drainage. To remove the remaining blood, perfusion was started with $0.9 \%(\mathrm{w} / \mathrm{v})$ sodium chloride solution at $37{ }^{\circ} \mathrm{C}$ for $1 \mathrm{~min}$. Retrograde perfusion was then performed using fixation solution ( $4 \%(\mathrm{w} / \mathrm{v})$ paraformaldehyde and $0.05 \%(\mathrm{v} / \mathrm{v})$ glutaraldehyde in $0.1 \mathrm{M}$ cacodylate buffer (0.1 M sodium cacodylate in $\mathrm{H}_{2} \mathrm{O}_{\text {bidest }}$ pH 7.4; $\mathrm{CB})$ ) at RT for $5 \mathrm{~min}$ [41]. After in situ MRI scans, the ascending aorta, the aortic arch (including brachiocephalic trunk, left common carotid artery and left subclavian artery), and the descending thoracic aorta were prepared and embedded in $1 \%(\mathrm{w} / \mathrm{v})$ low melting point agarose (Thermo Fisher Scientific, Waltham, MA) in a $15 \mathrm{~mL}$ Falcon $^{\circledR}$ tube for ex vivo MRI scans.

MRI analyses were performed on a 7 Tesla BioSpec 70/20 USR scanner (Bruker, Billerica, MA) equipped with a $1 \mathrm{H}$-CryoProbe coil (Bruker). First, in situ MRI scans of retrogradely perfused animals were recorded. FLASH 2D overviews were acquired with the following parameters: field of view $(\mathrm{FOV})=25 \times 25 \mathrm{~mm}$; repetition time (TR)/echo time $(\mathrm{TE})=250 \mathrm{~ms} / 3.5 \mathrm{~ms}$, flip angle $(\mathrm{FA})=30^{\circ}, 6$ averages (NEX), matrix dimension
(MD) $256 \times 256,20$ consecutive $0.3 \mathrm{~mm}$ thick slices, acquisition time (TA) $=4 \mathrm{~min}$ and $18 \mathrm{~s}$, followed by FLASH 3D scans: FOV $=19.2 \times 19.2 \times 16 \mathrm{~mm}$; TR/TE $=$ $36.5 \mathrm{~ms} / 8 \mathrm{~ms}, \mathrm{FA}=20^{\circ}, \mathrm{NEX}=2, \mathrm{MD} 192 \times 192 \times 160$; $\mathrm{TA}=42 \mathrm{~min}$ and $2 \mathrm{~s}$.

To evaluate the uptake of VSOPs into atherosclerotic plaques at various time points after intravenous injection, semiquantitative analyses of MRI scans of the aortic arch were performed ex vivo using a FLASH 3D pulse sequence: $\mathrm{FOV}=19.2 \mathrm{~mm}^{3}$, TR/TE $=31 \mathrm{~ms} / 8 \mathrm{~ms}$, $\mathrm{FA}=20^{\circ}, \mathrm{NEX}=1, \mathrm{MD} 192 \times 192 \times 192 ; \mathrm{TA}=21 \mathrm{~min}$ and $25 \mathrm{~s}$. VSOP accumulation causes signal extinctions in $2^{*}$ sequences. Thus, dark voxels, having less than $67 \%$ signal magnitude compared to the surrounding agarose, were counted in cross-sections of the ascending aorta to determine the percentage of dark voxels within the vessel wall. Five consecutive sections were analyzed in 1 animal per time point after VSOP injection. Image processing and analyses were performed using ImageJ (http://imagej.nih.gov/ij/).

\subsection{TEM}

Animals were sacrificed under isoflurane general anesthesia by blood withdrawal and subsequent perfusion via the left ventricle with drainage over the right atrium. To remove the remaining blood, perfusion was started with $0.9 \%(\mathrm{w} / \mathrm{v})$ sodium chloride solution at $37^{\circ} \mathrm{C}$ for $1 \mathrm{~min}$ at a pressure of $100 \mathrm{mmHg}$. Thereafter, animals were perfused with fixation solution consisting of $4 \%(\mathrm{w} / \mathrm{v})$ paraformaldehyde and $0.05 \%(\mathrm{v} / \mathrm{v})$ glutaraldehyde in $0.1 \mathrm{M} \mathrm{CB}$ at RT for $5 \mathrm{~min}$ at a pressure of $100 \mathrm{mmHg}$ [41]. The ascending aorta, the aortic arch (including brachiocephalic trunk, left common carotid artery, and left subclavian artery), and the descending thoracic aorta were prepared and post-fixed in a solution of $4 \%(\mathrm{w} / \mathrm{v})$ paraformaldehyde and $4 \%(\mathrm{v} / \mathrm{v})$ glutaraldehyde in $0.1 \mathrm{M} \mathrm{CB}$ at $\mathrm{pH} 7.4$ at RT for $1 \mathrm{~h}$. After fixation, samples were washed in $\mathrm{CB}$ containing $0.15 \mathrm{M}$ sucrose at $\mathrm{pH} 7.4$ for $3 \times 5 \mathrm{~min}$. Thereafter, samples were treated with $4 \%(\mathrm{w} / \mathrm{v})$ osmium tetroxide in $\mathrm{H}_{2} \mathrm{O}_{\text {bidest }}$ for $60 \mathrm{~min}$ at $\mathrm{RT}$ and subsequently washed again in $0.1 \mathrm{M} \mathrm{CB}$ for $3 \times 10 \mathrm{~min}$. Samples were dehydrated in ethanol $(70 \%, 80 \%, 90 \%$, $96 \%$, and $100 \%(\mathrm{v} / \mathrm{v}) ; 2 \times 10 \mathrm{~min}$ each), transferred into $100 \%$ propylene oxide $(2 \times 30 \mathrm{~min})$, and embedded 
in epoxy embedding medium according to the manufacturer's instructions (Sigma-Aldrich). Resin was polymerized for $24 \mathrm{~h}$ at $60^{\circ} \mathrm{C}$. Ultrathin sections $(60 \mathrm{~nm})$ were cut using an ultramicrotome (Leica, Wetzlar, Germany) with a diamond knife (Diatome, Hatfield, PA). Ultrathin sections were collected on 300-mesh copper grids (Plano, Lunen, Germany). TEM was performed on an EM 912 instrument (Carl Zeiss, Oberkochen, Germany) [42].

\subsection{Post-contrasting of ultrathin sections}

For better visualization of the ultrastructure, individual grids underwent post-contrasting. These grids were incubated in droplets of $2 \%(\mathrm{w} / \mathrm{v})$ uranyl acetate (Merck, Kenilworth, NJ) in $70 \%(\mathrm{v} / \mathrm{v})$ ethanol on Parafilm ${ }^{\circledR}$ for 2 min in the dark. Afterwards, grids were jet-rinsed with $\mathrm{H}_{2} \mathrm{O}_{\text {bidest }}$ twice and air-dried again. Sections were post-contrasted with lead citrate (133 mg lead nitrate, $200 \mathrm{mg}$ sodium citrate, and $80 \mu \mathrm{L}$ $10 \mathrm{~N}$ aqueous sodium hydroxide in $5 \mathrm{~mL} \mathrm{H}_{2} \mathrm{O}_{\text {bidest }}$ ) for $20 \mathrm{~s}$, again followed by 2 jet-rinsing steps with $\mathrm{H}_{2} \mathrm{O}_{\text {bidest }}$ and air-drying [42].

\subsection{Preparation of PMBCs and RBCs}

PBMCs and RBCs were isolated from the blood sample by density-gradient centrifugation with lympholyte- $\mathrm{M}^{\circledR}$ (Cedarlane, Burlington, ON, Canada). PBMCs and RBCs were washed with phosphate-buffered saline (PBS) and sedimented by centrifugation. For TEM analysis, the cell pellets were fixed in $4 \%(\mathrm{w} / \mathrm{v})$ paraformaldehyde and $4 \%(\mathrm{v} / \mathrm{v})$ glutaraldehyde in $0.1 \mathrm{M} \mathrm{CB}$ at $\mathrm{pH} 7.4$ at RT for $1 \mathrm{~h}$ and further processed for TEM analysis as described above. For ex vivo uptake studies, PBMCs and RBCs were counted and $3.35 \times 10^{5}$ cells were incubated with $0.75 \mathrm{mM}$ VSOPs in $100 \mu \mathrm{L}$ PBS in polymerase chain reaction tubes $(0.2 \mathrm{~mL})$ at $37^{\circ} \mathrm{C}$ for the indicated times in duplicates. Following incubation, the cells were centrifuged at $1,200 \mathrm{~g}$ for $5 \mathrm{~min}$ and washed 3 times with PBS. Total superparamagnetic iron content was measured by MPS.

\subsection{MPS}

MPS measurements of VSOP content in organs and blood were performed using a commercial magnetic particle spectrometer (Bruker) as previously described in detail [31]. VSOP calibration curves had high correlation coefficients $\left(R^{2}=0.99\right)$ and the limit of detection with a signal-to-noise ratio of 3 was assessed as $91 \mathrm{ng}$, reflecting the high sensitivity of this method.

\subsection{Cell culture and treatments}

HUVECs were isolated by collagenase type II (Merck) digestion of human umbilical veins as described previously [43]. HUVECs were cultured in EC medium (MCDB 131 media, Gibco ${ }^{\circledR}$, Thermo Fisher Scientific), supplemented with $2 \%(\mathrm{v} / \mathrm{v})$ fetal calf serum, $0.5 \mu \mathrm{L} \cdot \mathrm{mL}^{-1}$ basic fibroblast growth factor (Biomol, Hamburg, Germany), $5 \mathrm{U} \cdot \mathrm{mL}^{-1}$ heparin (Merck), $0.1 \mathrm{ng} \cdot \mathrm{mL}^{-1}$ epidermal growth factor (Biomol), $1 \mu \mathrm{g} \cdot \mathrm{mL}^{-1}$ hydrocortisone, $10 \mu \mathrm{L} \cdot \mathrm{mL}^{-1}$ streptomycin (Thermo Fisher Scientific), $10 \mu \mathrm{L} \cdot \mathrm{mL}^{-1} \mathrm{~L}$-glutamine (Thermo Fisher Scientific), and $4 \mu \mathrm{L} \cdot \mathrm{mL}^{-1}$ endothelial cell growth supplement (Promocell, Heidelberg, Germany) in a humidified incubator at $37^{\circ} \mathrm{C}$ with $5 \% \mathrm{CO}_{2}$. HUVECs were used until passage 5 . Uptake studies under different flow conditions were performed using the ibidi unidirectional laminar flow pump system (ibidi, Planegg, Germany). A total of 150,000 HUVECs were seeded in IbiTreat $\mu$-slides I 0.8 Luer or IbiTreat $\mu$-slides Y-shaped (ibidi) and incubated overnight under static conditions to allow the cells to adhere and grow to confluence. Cell culture was continued under static or flow conditions $\left(10 \mathrm{dyn} \cdot \mathrm{cm}^{-2}\right)$ for $48 \mathrm{~h}$ ( $\mu$-slides Y-shaped) or $72 \mathrm{~h}$ ( $\mu$-slides I 0.8 Luer). Where indicated, cells were stimulated with $5 \mathrm{ng} \cdot \mathrm{mL}^{-1}$ TNF $\alpha$ for $24 \mathrm{~h}$ prior to VSOP administration. After 48 or $72 \mathrm{~h}$, HUVECs were incubated with $0.75 \mathrm{mM}$ VSOPs under flow conditions $\left(10 \mathrm{dyn} \cdot \mathrm{cm}^{-2}\right)$ for $3 \mathrm{~h}$. For endocytosis experiments, HUVECs were plated onto gelatin-coated LabTek ${ }^{\mathrm{TM}}$ chambered glass coverslips (Nunc, Roskilde, Denmark) $6 \mathrm{~h}$ before the experiment. HUVECs that were pretreated with siRNA or dynasore as described below were incubated with VSOPs $(0.15 \mathrm{mM})$ for $60 \mathrm{~min}$ at $37^{\circ} \mathrm{C}$ in EC medium.

\subsection{Inhibition of endocytosis}

For pharmacological inhibition of endocytosis, HUVECs were pretreated before VSOP application with 75 or $150 \mu \mathrm{M}$ dynasore (Abcam, Cambridge, UK) 
for $30 \mathrm{~min}$ at $37^{\circ} \mathrm{C}$. Efficacy of inhibition was tested by analyzing the uptake of the fluorescently labeled endocytosis markers hTF $\left(12 \mu \mathrm{g} \cdot \mathrm{mL}^{-1}\right.$ hTF-FITC; Molecular Probes, Eugene, OR) and BSA $\left(2.5 \mu \mathrm{g} \cdot \mathrm{mL}^{-1}\right.$ BSA-Texas Red; Molecular Probes) after $60 \mathrm{~min}$ incubation in EC medium by fluorescence microscopy using a Zeiss Axiovert microscope connected to a Zeiss AxioCam MrC. Knockdown of human CAV-1 and human clathrin heavy chain (CLTC) expression was achieved using Silencer ${ }^{\circledR}$ Select validated siRNA ID: s2446 and siRNA ID: s475 (Ambion ${ }^{\circledR}$, Thermo Fisher Scientific), respectively. Silencer ${ }^{\circledR}$ Select negative control siRNA (Thermo Fisher Scientific) served as a control. Cells were seeded onto 6-well plates and cultured for $24 \mathrm{~h}$ to reach $50 \%-60 \%$ confluency on the day of transfection. Oligofectamine ${ }^{\mathrm{TM}}$ (Invitrogen, Carlsbad, CA) was used for transfection according to the manufacturer's protocol. An initial transfection was followed by a second transfection after $24 \mathrm{~h}$. Cells were plated onto gelatin-coated LabTek ${ }^{\mathrm{TM}}$ chambered glass coverslips (Nunc) $42 \mathrm{~h}$ after the second transfection and allowed to adhere for $6 \mathrm{~h}$ before VSOP incubation.

\subsection{Western blot analysis}

Following transfection, cells were washed twice with PBS and lysed in extraction buffer $(50 \mathrm{mM}$ Tris- $\mathrm{HCl}, 150 \mathrm{mM} \mathrm{KCl}, 5 \mathrm{mM}$ glucose, $0.5 \mathrm{mM}$ ethylenediaminetetraacetic acid $\mathrm{pH}$ 8.0, $0.5 \mathrm{mM}$ phenylmethylsulfonyl fluoride, $2 \mathrm{mM}$ dithiothreitol, and $1 \%(\mathrm{v} / \mathrm{v})$ Triton $\left.^{\mathrm{TM}} \mathrm{X}-100\right)$. Total protein $(5 \mu \mathrm{g}$ per lane) was subjected to sodium dodecyl sulfate polyacrylamide gel electrophoresis, followed by transfer to nitrocellulose membranes. Membranes were probed with anti-CAV-1 (N-20) or anti CLTC (C-20) antibodies (Santa Cruz Biotechnology, Dallas, TX). This was followed by incubation with secondary antibodies conjugated with horseradish peroxidase and detection with ECL Plus (GE Healthcare, Little Chalfont, UK). Amido black staining of membranes served as a control for equal protein loading.

\subsection{Quantification of VSOP uptake in HUVECs}

After VSOP incubation, cells were gently washed with PBS and fixed with $4 \%(\mathrm{w} / \mathrm{v})$ paraformaldehyde
(Roti ${ }^{\circledR}$-Histofix 4\%, Carl Roth, Karlsruhe, Germany) for $10 \mathrm{~min}$. Cells were rinsed with PBS and stained with Prussian blue $(2 \%(\mathrm{w} / \mathrm{v})$ potassium ferrocyanide in $1 \%(\mathrm{v} / \mathrm{v}) \mathrm{HCl})$ for $5 \mathrm{~min}$. Where indicated, staining with Prussian blue was followed by counterstaining with nuclear fast red (Carl Roth) for $5 \mathrm{~min}$. After staining, the ibiTreat $\mu$-slides were washed with PBS and filled with mounting medium for microscopy (Thermo Fisher Scientific). Six images per slide at predefined regions of interest were immediately acquired using a Zeiss Axiovert microscope connected to Zeiss AxioCam MrC. Images were analyzed using Zeiss AxioVision software and VSOP content was calculated as the percentage Prussian blue-stained area. For endocytosis experiments, the Prussian bluestained area per cell was estimated using AxioVision software. The mean area per cell was averaged from 6 regions of interest per chamber.

\subsection{Data analyses}

Data are presented as mean \pm standard deviation. Paired Student's $t$-test or one-way analysis of variance with Tukey's multiple comparison test was used where appropriate. $p$-values of $<0.05$ were considered to be statistically significant. Statistics were calculated using Prism 6 (GraphPad, La Jolla, CA).

\section{Acknowledgements}

The project was supported by the Deutsche Forschungsgemeinschaft (DFG) within the Clinical Research Unit KFO 213 (Nos. STA 481/1-2, LU 1559/1-2, TA 166/7-2, and WA 3105/1-2) and the Bundesministerium für Bildung und Forschung (BMBF) (No. DZHK B15-028). Additional funding was provided by the DFG (EXC Neurocure) and the BMBF (01EO0801, Center for Stroke Research Berlin) and 01EW1201 under the ERA-NET-NEURON scheme funded by the European Commission (PBS). We highly appreciate the excellent technical assistance of A. Stach, S. Metzkow, and M. Andratzek. ES provided the molecular model of VSOP in the graphical abstract. WCP is participant in the BIH-Charité Clinical Scientist Program funded by the Charité-Universitätsmedizin Berlin and the Berlin Institute of Health. 


\section{References}

[1] Mozaffarian, D.; Benjamin, E. J.; Go, A. S.; Arnett, D. K.; Blaha, M. J.; Cushman, M.; de Ferranti, S.; Després, J. P.; Fullerton, H. J.; Howard, V. J. et al. Heart disease and stroke statistics-2015 update: A report from the American Heart Association. Circulation 2015, 131, e29-e322.

[2] Davignon, J.; Ganz, P. Role of endothelial dysfunction in atherosclerosis. Circulation 2004, 109, III27-III32.

[3] Libby, P.; Ridker, P. M.; Hansson, G. K. Progress and challenges in translating the biology of atherosclerosis. Nature 2011, 473, 317-325.

[4] Bonetti, P. O.; Lerman, L. O.; Lerman, A. Endothelial dysfunction: A marker of atherosclerotic risk. Arterioscler. Thromb. Vasc. Biol. 2003, 23, 168-175.

[5] Fleg, J. L.; Stone, G. W.; Fayad, Z. A.; Granada, J. F.; Hatsukami, T. S.; Kolodgie, F. D.; Ohayon, J.; Pettigrew, R.; Sabatine, M. S.; Tearney, G. J. et al. Detection of high-risk atherosclerotic plaque: Report of the NHLBI Working Group on current status and future directions. JACC Cardiovasc. Imaging 2012, 5, 941-955.

[6] Nörenberg, D.; Ebersberger, H. U.; Diederichs, G.; Hamm, B.; Botnar, R. M.; Makowski, M. R. Molecular magnetic resonance imaging of atherosclerotic vessel wall disease. Eur. Radiol. 2016, 26, 910-920.

[7] Makowski, M. R.; Wiethoff, A. J.; Blume, U.; Cuello, F.; Warley, A.; Jansen, C. H. P.; Nagel, E.; Razavi, R.; Onthank, D. C.; Cesati, R. R. et al. Assessment of atherosclerotic plaque burden with an elastin-specific magnetic resonance contrast agent. Nat. Med. 2011, 17, 383-388.

[8] Chen, W.; Cormode, D. P.; Vengrenyuk, Y.; Herranz, B.; Feig, J. E.; Klink, A.; Mulder, W. J. M.; Fisher, E. A.; Fayad, Z. A. Collagen-specific peptide conjugated HDL nanoparticles as MRI contrast agent to evaluate compositional changes in atherosclerotic plaque regression. JACC Cardiovasc. Imaging 2013, 6, 373-384.

[9] Makowski, M. R.; Forbes, S. C.; Blume, U.; Warley, A.; Jansen, C. H. P.; Schuster, A.; Wiethoff, A. J.; Botnar, R. M. In vivo assessment of intraplaque and endothelial fibrin in $\mathrm{ApoE}^{-/-}$mice by molecular MRI. Atherosclerosis 2012, 222, 43-49.

[10] Phinikaridou, A.; Andia, M. E.; Protti, A.; Indermuehle, A.; Shah, A.; Smith, A.; Warley, A.; Botnar, R. M. Noninvasive magnetic resonance imaging evaluation of endothelial permeability in murine atherosclerosis using an albuminbinding contrast agent. Circulation 2012, 126, 707-719.

[11] Ruehm, S. G.; Corot, C.; Vogt, P.; Kolb, S.; Debatin, J. F. Magnetic resonance imaging of atherosclerotic plaque with ultrasmall superparamagnetic particles of iron oxide in hyperlipidemic rabbits. Circulation 2001, 103, 415-422.
[12] Kooi, M. E.; Cappendijk, V. C.; Cleutjens, K. B. J. M.; Kessels, A. G. H.; Kitslaar, P. J. E. H. M.; Borgers, M.; Frederik, P. M.; Daemen, M. J. A. P.; van Engelshoven, J. M. A. Accumulation of ultrasmall superparamagnetic particles of iron oxide in human atherosclerotic plaques can be detected by in vivo magnetic resonance imaging. Circulation 2003, 107, 2453-2458.

[13] Taupitz, M.; Wagner, S.; Schnorr, J.; Kravec, I.; Pilgrimm, H.; Bergmann-Fritsch, H.; Hamm, B. Phase I clinical evaluation of citrate-coated monocrystalline very small superparamagnetic iron oxide particles as a new contrast medium for magnetic resonance imaging. Invest. Radiol. 2004, 39, 394-405.

[14] Wagner, M.; Wagner, S.; Schnorr, J.; Schellenberger, E.; Kivelitz, D.; Krug, L.; Dewey, M.; Laule, M.; Hamm, B.; Taupitz, M. Coronary MR angiography using citrate-coated very small superparamagnetic iron oxide particles as bloodpool contrast agent: Initial experience in humans. J. Magn. Reson. Imaging 2011, 34, 816-823.

[15] Ludwig, A.; Poller, W. C.; Westphal, K.; Minkwitz, S.; Lättig-Tünnemann, G.; Metzkow, S.; Stangl, K.; Baumann, G.; Taupitz, M.; Wagner, S. et al. Rapid binding of electrostatically stabilized iron oxide nanoparticles to THP-1 monocytic cells via interaction with glycosaminoglycans. Basic Res. Cardiol. 2013, 108, 328.

[16] Makowski, M. R.; Varma, G.; Wiethoff, A. J.; Smith, A.; Mattock, K.; Jansen, C. H. P.; Warley, A.; Taupitz, M.; Schaeffter, T.; Botnar, R. M. Noninvasive assessment of atherosclerotic plaque progression in $\mathrm{ApoE}^{-/-}$mice using susceptibility gradient mapping. Circ. Cardiovasc. Imaging 2011, 4, 295-303.

[17] Scharlach, C.; Kratz, H.; Wiekhorst, F.; Warmuth, C.; Schnorr, J.; Genter, G.; Ebert, M.; Mueller, S.; Schellenberger, E. Synthesis of acid-stabilized iron oxide nanoparticles and comparison for targeting atherosclerotic plaques: Evaluation by MRI, quantitative MPS, and TEM alternative to ambiguous Prussian blue iron staining. Nanomedicine 2015, 11, 10851095.

[18] Wagner, S.; Schnorr, J.; Ludwig, A.; Stangl, V.; Ebert, M.; Hamm, B.; Taupitz, M. Contrast-enhanced MR imaging of atherosclerosis using citrate-coated superparamagnetic iron oxide nanoparticles: Calcifying microvesicles as imaging target for plaque characterization. Int. J. Nanomedicine 2013, $8,767-779$

[19] Makowski, M. R.; Henningsson, M.; Spuentrup, E.; Kim, W. Y.; Maintz, D.; Manning, W. J.; Botnar, R. M. Characterization of coronary atherosclerosis by magnetic resonance imaging. Circulation 2013, 128, 1244-1255.

[20] Zimmerman, M.; McGeachie, J. Quantitation of the relationship between aortic endothelial intercellular cleft morphology and permeability to albumin. Atherosclerosis 
1986, 59, 277-282.

[21] Gerrity, R. G.; Richardson, M.; Somer, J. B.; Bell, F. P.; Schwartz, C. J. Endothelial cell morphology in areas of in vivo Evans blue uptake in the aorta of young pigs. II. Ultrastructure of the intima in areas of differing permeability to proteins. Am. J. Pathol. 1977, 89, 313-334.

[22] Sigovan, M.; Boussel, L.; Sulaiman, A.; Sappey-Marinier, D.; Alsaid, H.; Desbleds-Mansard, C.; Ibarrola, D.; Gamondes, D.; Corot, C.; Lancelot, E. et al. Rapid-clearance iron nanoparticles for inflammation imaging of atherosclerotic plaque: Initial experience in animal model. Radiology 2009, 252, 401-409.

[23] Schekman, R.; Singer, S. J. Clustering and endocytosis of membrane receptors can be induced in mature erythrocytes of neonatal but not adult humans. Proc. Natl. Acad. Sci. USA 1976, 73, 4075-4079.

[24] Moreno, P. R.; Purushothaman, K. R.; Sirol, M.; Levy, A. P.; Fuster, V. Neovascularization in human atherosclerosis. Circulation 2006, 113, 2245-2252.

[25] Macia, E.; Ehrlich, M.; Massol, R.; Boucrot, E.; Brunner, C.; Kirchhausen, T. Dynasore, a cell-permeable inhibitor of dynamin. Dev. Cell 2006, 10, 839-850.

[26] Lobatto, M. E.; Calcagno, C.; Millon, A.; Senders, M. L.; Fay, F.; Robson, P. M.; Ramachandran, S.; Binderup, T.; Paridaans, M. P. M.; Sensarn, S. et al. Atherosclerotic plaque targeting mechanism of long-circulating nanoparticles established by multimodal imaging. ACS Nano 2015, 9, 1837-1847.

[27] Vasile, E.; Simionescu, M.; Simionescu, N. Visualization of the binding, endocytosis, and transcytosis of low-density lipoprotein in the arterial endothelium in situ. J. Cell Biol. 1983, 96, 1677-1689.

[28] Kühn, M. W. M.; Armstrong, S. A. Designed to kill: Novel menin-MLL inhibitors target MLL-rearranged leukemia. Cancer Cell 2015, 27, 431-433.

[29] Zhou, J.; Li, Y. S.; Chien, S. Shear stress-initiated signaling and its regulation of endothelial function. Arterioscler. Thromb. Vasc. Biol. 2014, 34, 2191-2198.

[30] Kolářová, H.; Ambrůzová, B.; Svihálková Šindlerová, L.; Klinke, A.; Kubala, L. Modulation of endothelial glycocalyx structure under inflammatory conditions. Mediators Inflamm. 2014, 2014, Article ID 694312.

[31] Poller, W. C.; Löwa, N.; Wiekhorst, F.; Taupitz, M.; Wagner, S.; Möller, K.; Baumann, G.; Stangl, V.; Trahms, L.; Ludwig, A. Magnetic particle spectroscopy reveals dynamic changes in the magnetic behavior of very small superparamagnetic iron oxide nanoparticles during cellular uptake and enables determination of cell-labeling efficacy. J. Biomed. Nanotechnol. 2016, 12, 337-346.
[32] Poon, G. M. K.; Gariépy, J. Cell-surface proteoglycans as molecular portals for cationic peptide and polymer entry into cells. Biochem. Soc. Trans. 2007, 35, 788-793.

[33] Zhang, S. Y.; Moustafa, Y.; Huo, Q. Different interaction modes of biomolecules with citrate-capped gold nanoparticles. ACS Appl. Mater. Interfaces 2014, 6, 21184-21192.

[34] Bai, K.; Wang, W. Shear stress-induced redistribution of the glycocalyx on endothelial cells in vitro. Biomech. Model. Mechanobiol. 2014, 13, 303-311.

[35] Zeng, Y.; Waters, M.; Andrews, A.; Honarmandi, P.; Ebong, E. E.; Rizzo, V.; Tarbell, J. M. Fluid shear stress induces the clustering of heparan sulfate via mobility of glypican-1 in lipid rafts. Am. J. Physiol. Heart Circ. Physiol. 2013, 305, H811-H820.

[36] Cruz-Chu, E. R.; Malafeev, A.; Pajarskas, T.; Pivkin, I. V.; Koumoutsakos, P. Structure and response to flow of the glycocalyx layer. Biophys. J. 2014, 106, 232-243.

[37] Samuel, S. P.; Jain, N.; O'Dowd, F.; Paul, T.; Kashanin, D.; Gerard, V. A.; Gun'ko, Y. K.; Prina-Mello, A.; Volkov, Y. Multifactorial determinants that govern nanoparticle uptake by human endothelial cells under flow. Int. J. Nanomedicine 2012, 7, 2943-2956.

[38] Matuszak, J.; Zaloga, J.; Friedrich, R. P.; Lyer, S.; Nowak, J.; Odenbach, S.; Alexiou, C.; Cicha, I. Endothelial biocompatibility and accumulation of SPION under flow conditions. J. Magn. Magn. Mater. 2015, 380, 20-26.

[39] Lin, A.; Sabnis, A.; Kona, S.; Nattama, S.; Patel, H.; Dong, J. F.; Nguyen, K. T. Shear-regulated uptake of nanoparticles by endothelial cells and development of endothelial-targeting nanoparticles. J. Biomed. Mater. Res. A 2010, 93, 833-842.

[40] Howard, M.; Zern, B. J.; Anselmo, A. C.; Shuvaev, V. V.; Mitragotri, S.; Muzykantov, V. Vascular targeting of nanocarriers: Perplexing aspects of the seemingly straightforward paradigm. ACS Nano 2014, 8, 4100-4132.

[41] Poller, W. C.; Bernard, R.; Derst, C.; Weiss, T.; Madai, V. I.; Veh, R. W. Lateral habenular neurons projecting to rewardprocessing monoaminergic nuclei express hyperpolarizationactivated cyclic nucleotid-gated cation channels. Neuroscience 2011, 193, 205-216.

[42] Poller, W. C.; Madai, V. I.; Bernard, R.; Laube, G.; Veh, R. W. A glutamatergic projection from the lateral hypothalamus targets VTA-projecting neurons in the lateral habenula of the rat. Brain Res. 2013, 1507, 45-60.

[43] Stangl, V.; Günther, C.; Jarrin, A.; Bramlage, P.; Moobed, M.; Staudt, A.; Baumann, G.; Stangl, K.; Felix, S. B. Homocysteine inhibits TNF- $\alpha$-induced endothelial adhesion molecule expression and monocyte adhesion via nuclear factor- $\kappa \mathrm{B}$ dependent pathway. Biochem. Biophys. Res. Commun. 2001, 280, 1093-1100. 